\title{
BYOE: Mobile Experiment for Signals and Systems - Analysis of a Guitar String
}

\section{Dr. Bonnie Ferri, Georgia Institute of Technology}

Bonnie Ferri is a Professor and Associate Chair for Undergraduate Affairs in the School of Electrical and Computer Engineering at Georgia Tech. She received her BS in EE from Notre Dame, her MS in MAE from Princeton, and her PhD in EE from Georgia Tech. She spent two years working for Honeywell, Inc. as a controls engineer. She has spent 10 years working on hands-on education and has won several awards including the Harriet B. Rigas Award from the IEEE Education Society.

\section{Dr. Aldo A. Ferri, Georgia Institute of Technology}

Dr. Ferri received his BS in Mechanical Engineering from Lehigh University in 1981 and his PhD in Mechanical and Aerospace Engineering from Princeton University in 1985. He has been a faculty member in the School of Mechanical Engineering at Georgia Tech since 1985 and currently serves as the Associate Chair for Undergraduate Studies in Mechanical Engineering. He is a Member of ASEE and a Fellow of ASME.

\section{Prof. Kenneth A Connor, Rensselaer Polytechnic Institute}

Kenneth Connor is a professor in the Department of Electrical, Computer, and Systems Engineering, where he teaches courses on plasma physics, electromagnetics, electronics and instrumentation, electric power, and general engineering. His research involves plasma physics, electromagnetics, photonics, engineering education, diversity in the engineering workforce, and technology enhanced learning. Since joining the Rensselaer faculty in 1974, he has been continuously involved in research programs at such places as Oak Ridge National Laboratory and the Universities of Texas and Wisconsin in the U.S., Kyoto and Nagoya Universities in Japan, the Ioffe Institute in Russia, and Kharkov Institute of Physics and Technology in Ukraine. He was ECSE Department Head from 2001-2008 and served on the board of the ECE Department Heads Association from 2003-2008. He is presently the Education Director for the SMART LIGHTING NSF ERC. 


\title{
BYOE: Mobile Experiment for Signals and Systems Courses:
}

\author{
Analysis of a Guitar String
}

\begin{abstract}
This paper presents an inexpensive, mobile experimental platform suitable for electronics, signals and systems, and vibrations courses. Two versions of the platform have been developed, one that is simpler and cheaper but less flexible with regard to the set of experiments that can be done on it. Both platforms have a single guitar string and a transducer to pick up the string vibration. The platforms rely on student data acquisition boards, for measuring the signals, and student laptops, for displaying the signals.
\end{abstract}

\section{Introduction}

Electric guitars are fascinating to students and provide an excellent motivating platform for engineering exploration in acoustics, vibration, electronics, electromagnetics, stresses and strain, and signals and systems ${ }^{1}$. Students generally respond very positively to the use of the guitar as a source of electrical signals because it is something they already know about, and they can physically see the creation of the signals when the guitar is plucked. Used as an experimental platform, a guitar provides sound, touch, and sight sensory input to students. Coupled with a data acquisition board, the electric guitar provides the source of a complex signal that might be analyzed in the time domain or in the frequency domain. The goal in this paper is to capture the main attributes of an electric guitar as an experimental platform but reduce the cost and improve the portability so that it can be used as a hands-on activity in lecture-based courses.

Lecture-based courses benefit from hands-on exposure to applications that demonstrate the theory taught in the classes. Experiments can reinforce and clarify the concepts, but most experiments are relegated to lab courses that are separate from the theoretical or lecture-based courses. It has been shown in other applications that cheap, portable experiments that can be done in class or at home by students can enhance the learning in lecture-based courses ${ }^{2-6}$. Recent advances in low-cost portable data acquisition boards, such as a Mobile Studio IOBboard $^{2}$ or a National Instrument $\operatorname{myDAQ}^{7}$, facilitate this type of pedagogy that more carefully integrates theory taught in traditional classes with experiments done traditionally in dedicated labs.

Some topics are more abstract than others, indicating a greater potential impact on student learning when using labs. Signals and systems concepts, especially, tend to be very mathematical and abstract, and students who prefer more practical or hands-on material are at a disadvantage in systems classes. A vibratory experiment that uses multiple senses, such as sight and sound, in addition to the analysis of measured data can help many students understand this topic better.

The experimental platform proposed in this paper consists of a guitar string mounted on a portable platform with a transducer to pick-up the vibration of the string. Two versions of the platform have been developed and have been used in electronics courses, K-12 outreach 
programs, and in signals and systems courses. The first version is simpler and cheaper but less flexible in terms of experiments that are possible.

Both versions of the platform rely on the use of student data acquisition boards and supporting software so that students can view the signals and perform analysis on the signals using their own laptops. The guitar string experiments highlighted in this paper have been implemented using the Mobile Studio IOBoard and the NI myDAQ data acquisition boards, both of which have analog input and audio output channels.

\section{Simple Guitar String Platform}

To demonstrate how an electric guitar works, a stripped down, single-string electric guitar was constructed with a metal guitar string, base, tuner, angle bracket, and home-made guitar pick-up. The platform, which is $38 \mathrm{~cm}$ long $x 5 \mathrm{~cm}$ wide $x 7.6 \mathrm{~cm}$ tall, is shown in Figure 1. The metal guitar string is pulled taut and connected to a lexan base through a fixed bracket on one end and a tuning peg on the other. The tuner is used to adjust the tension in the string. The transducer in this platform is made by winding a fine (high gauge) wire around a cylindrical Alnico post permanent magnet. This transducer acts as a guitar pick-up by mounting it just under the guitar string, close enough to induce current when metal guitar string vibrates. The home-made pickup has two advantages over a commercial guitar pick-up, cost (about \$2 versus \$20-\$30) and transparency to the underlying physical phenomena of the pick-up (generation of electromagnetic signals).

Complete devices were constructed or provided in kit form to build as part of a learning activity in K-12 outreach events and a circuits course. Winding a pickup and putting together the guitar has been particularly popular with high school teachers who appreciate knowing construction details for experiments they use in their classes.

In outreach events (e.g. the USA Science and Engineering Festival in 2012), electrical signals produced by the simple guitar string platform were displayed with the Mobile Studio data acquisition board, which also allowed participants to hear the sounds produced using ear buds driven by the audio amplifier on the board. Inquisitive children of all ages were able to pluck the string, see and hear the signal under a variety of conditions (string tightness, strength of pluck, etc.) and discuss what they experienced with their family and the college and high school students who assisted at the booth. Outreach also provides a good test of the physical robustness of the apparatus.

In an introductory Electric Circuits class for EE majors at Rensselaer Polytechnic Institute, the students were given a project to design a tone control circuit for their one string guitar. They were asked to sketch a Bode plot for a transfer function that would provide the desired tone control. Then they were to design an active or passive contour shaping circuit to achieve the desired result. The signal from the guitar pick-up was passed through their tone control circuit and then routed to the audio output jack on the Mobile Studio IOBoard. Again, because they were able to both see (on the 'scope) and hear the impact of their designs (through their earbuds from the audio jack), an amazing level of creativity was evident in the collection of work from the class of about 100 students. 


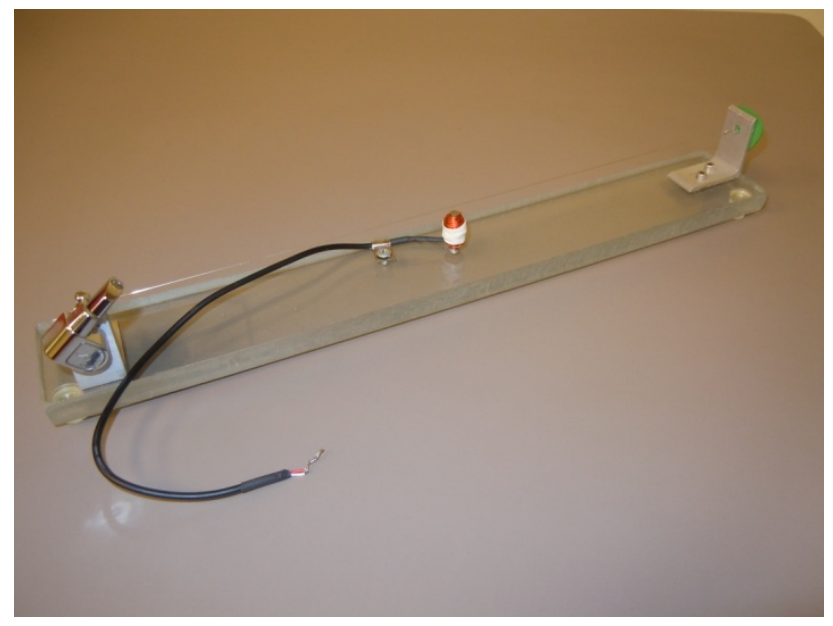

Figure 1: Simple guitar string experiment.

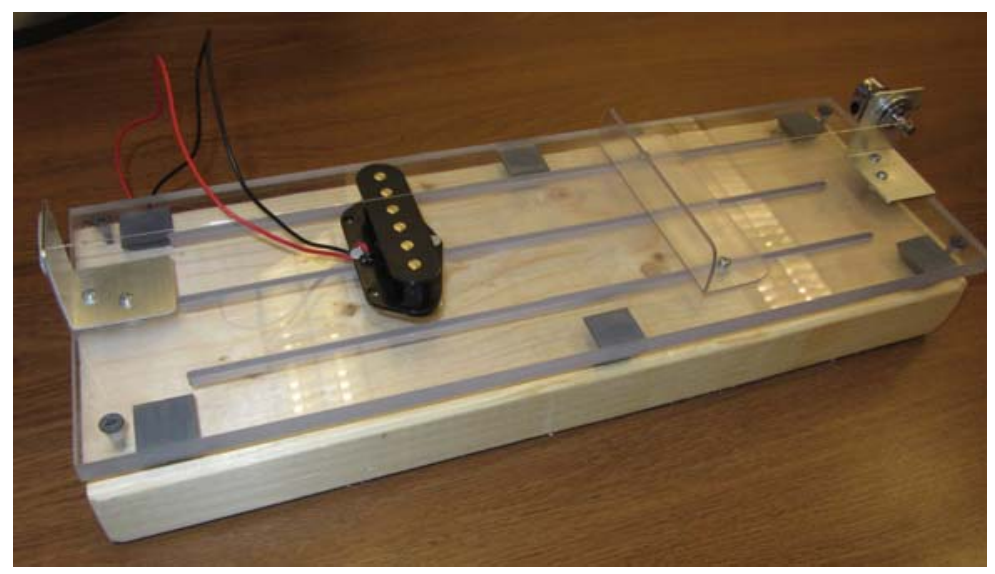

Figure 2: Complex guitar string platform.

\section{Complex Guitar String Platform and Experiment}

A more advanced version of the guitar string platform was designed to give more flexibility in the types of experiments possible and to improve the quality of the measured signal. The complex platform, shown in Figure 2, is $41 \mathrm{~cm}$ long $\times 10 \mathrm{~cm}$ wide $\times 7.6 \mathrm{~cm}$ tall. The basic construction is the same as the simple platform, with a single metal guitar string mounted on a base through a fixed bracket on one end and a tuning peg on the other end. The fundamental modifications are the addition of a movable fret and a movable base for the transducer. Other modifications, the use of a commercial guitar pick-up and a board to add weight, were done to improve the quality of the measured signal and to isolate the string vibration from the base vibration.

The transducer was put on a sliding base so that students can see the effect of transducer location on the signal, essentially, examining the observability of the system. A vibrating string has modes of vibration, which differ in shape and in frequency. See the appendix for a background discussion on the equations and the mode shapes for a vibrating string. Placing a transducer under the middle point of the string is the best position for measuring the first harmonic, but this 
position is at the node of the second harmonic of vibration. Hence, the second mode is unobservable for a sensor at that location. All of the modes are unobservable from transducers placed at their nodes. This unobservability phenomenon manifests itself by the relative height of the harmonic peaks in the frequency response.

Similarly, the controllability of the system can be explored by changing the location where the impulsive input is applied. Applying a force at a node of a harmonic does not excite that harmonic, so hitting the string in the middle does not excite the second harmonics very much. Students can compare the relative peaks of the first harmonic and the second harmonic when hitting the string in the middle versus hitting it near one of the endpoints. Note, controllability and observability may not be introduced in such an early class in the subject, but the concepts can still be explored, where the measured signal is missing some components depending on where the forcing term or where the transducer are located.

Students can compute the transfer function of the system from the differential equations and determine the effect of system properties such as the tension in the string and the length of the string. The tuning peg allows the tension to be changed, and the fret allows for the effective length of the string to be changed to obtain a desired fundamental harmonic frequency.

The experimental platform has been used in a lecture-based signals and systems class as an inclass experiment to be done by students at their desks working in two-person groups. The lab consists of a prelab where they compute the transfer function of the string and plot the frequency response. During lab, they use a screw driver shaft to impart an impulse to the system, and they also pluck the string to see the free response. They are asked to adjust system properties such as the tension in the string and the length of the string (by using a fret). A RockIt ${ }^{\text {TM }}$ speaker is used as a small, cheap vibration shaker to excite the system sinusoidally and to do a sine sweep.

The fundamental concepts explored in the lab are the relationship between time domain and frequency domain signal characteristics, harmonic content of signals, impulse response, initial condition response, calculating a transfer function from equations, calculating and plotting the frequency response from the model, frequency response from impulse response data and from sine sweeps, resonance and low damping in systems, and frequency response dependence on physical properties of the system. Details about this particular lab are discussed below.

The lab instructions contain a background section with necessary information that would not be contained in standard signals and systems lectures. This information includes the following text:

Examples of transducers - guitar pickups: A transducer is a device that converts a physical quantity into an electrical signal that can be measured. A guitar pickup is a transducer that converts vibration of a guitar string into a voltage signal. The pickup has a permanent magnet wrapped with a copper coil that creates a magnetic field. A metal string vibrating within that magnetic field induces an electrical signal that is proportional to the velocity of the vibration of the string at the point of measurement.

Dynamic Signal (Spectrum) Analyzer (DSA): A spectrum analyzer measures a signal from a transducer and computes the Fourier Transform of the signal, which is displayed on a graph of magnitude versus frequency. Internally, the spectrum analyzer uses the Fast Fourier Transform (FFT) of the sampled signal to compute an approximation to the exact Fourier Transform. Also, DSAs are often programmed to compute the Fourier Transform (using an FFT) on a window of data. This means that the DSA takes a window of data 
and computes the frequency spectrum on that window of data, displays it, and then takes a new window of data to repeat the process.

Vibrating strings: A string attached at both ends has a dynamic response when excited externally, such as being plucked like a guitar string or hit like a piano string. Since neither end of the string can move, the string vibrates following specific modal shapes with the amount of deflection dependent on the position along the string. For example, plucking the string in the middle will excite the string to vibrate in the shape of Mode 1, shown below, as well as the other odd-numbered modes.

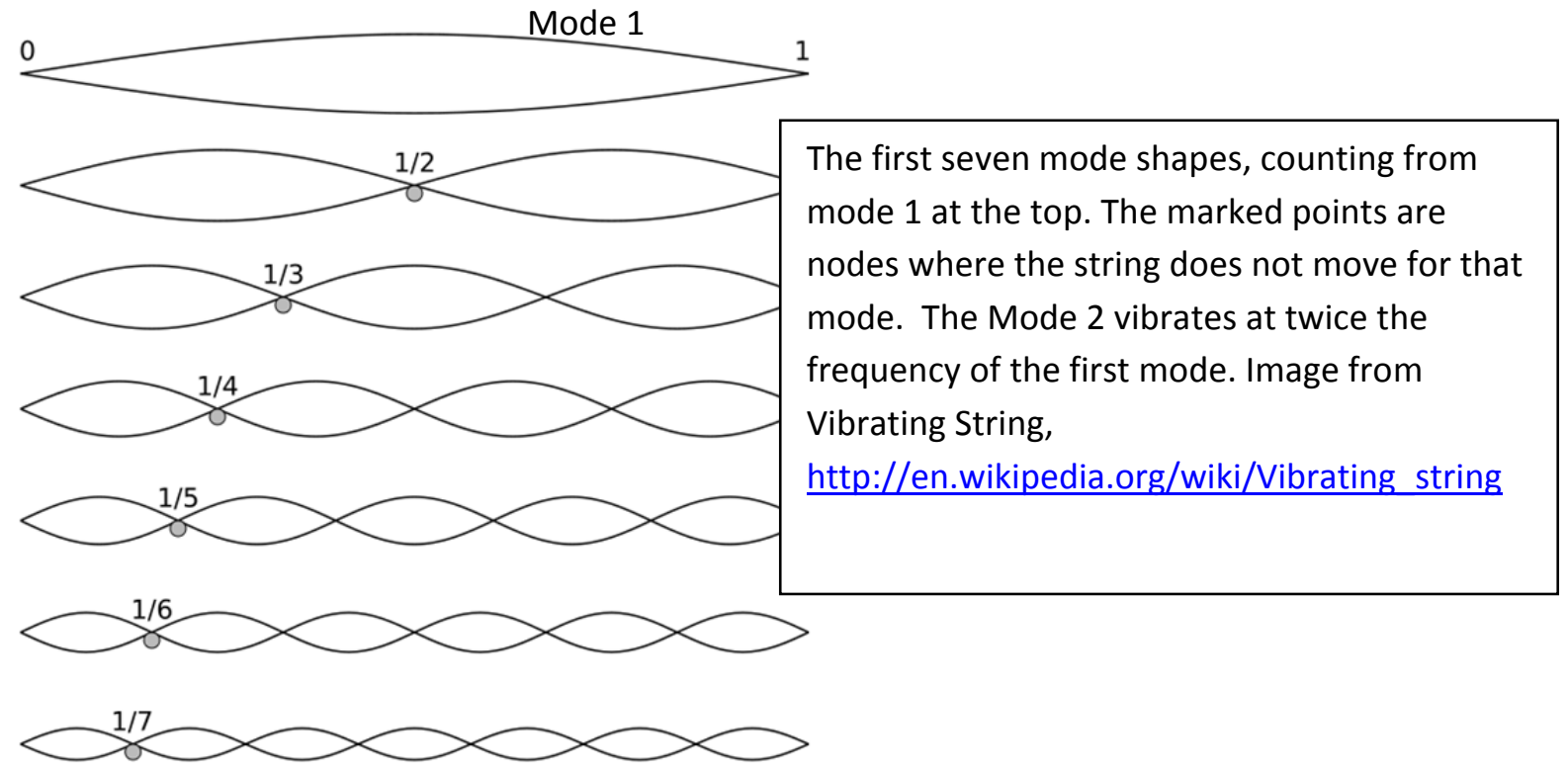

Figure 3: Mode shapes of a fixed-fixed string.

To determine the frequency (in Hertz) of each mode, use the expression

$$
f=\frac{k}{2 L} \sqrt{\frac{T}{\mu}}
$$

where $T$ is the tension in the string, $L$ is the string length, $\mu$ is the density of the string (mass per unit length), and $k$ is the mode number. The frequency can be tuned by increasing the tension or changing the length. In stringed musical instruments, such as a guitar, the tuner is used to tighten the string, i.e., change the tension. Frets are bars placed across the strings that effectively change the length of the string.

The fundamental frequency is the frequency of the first mode, $k=1$. Harmonics are the higher frequencies, so the second harmonic is the mode shape and frequency corresponding to $k=2$.

Free and forced response of a string: When you pluck a guitar string, you are momentarily giving it an initial condition on the displacement. The resulting response is a zero input response (also known as the free response) to an initial condition. Striking the string with a hammer, as in a piano, is equivalent to giving the string an impulse, so the resulting response is the impulse response (here the string starts at rest so this is the zero 
state response, or the forced response). In all cases, the different modes are excited and the response is a superposition of the modes. Details of the model are given in the Appendix.

Musical Notes: The table below gives the frequencies of some musical notes.

\begin{tabular}{|c|c|}
\hline Note & Frequency $(\mathrm{Hz})$ \\
\hline \hline C & 261.6 \\
\hline D & 293.7 \\
\hline E & 329.6 \\
\hline F & 349 \\
\hline G & 392 \\
\hline A & 440 \\
\hline B & 493.9 \\
\hline
\end{tabular}

Breadboards: Breadboards (also known as protoboards) make many connections under the board in order to reduce the number of wires that you have to connect. Typically, groups of 5 holes are

Each vertical group of 5 holes is connected.

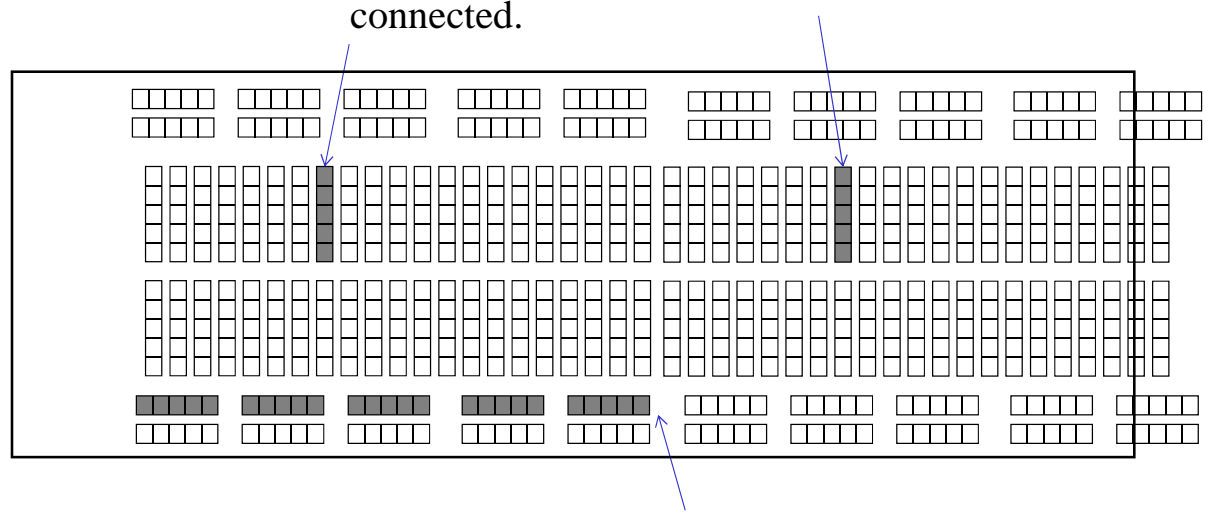

Every hole in this horizontal line is connected.

The prelab asks students to find an expression for the transfer function $\mathrm{Y}(\mathrm{s}) / \mathrm{U}(\mathrm{s})$ for the system from the equations in the Appendix, given the following system parameters: $\mathrm{L}=1 \mathrm{~m}, \mathrm{x}_{\mathrm{r}}=0.25$ $\mathrm{m}, \mathrm{x}_{\mathrm{f}}=0.1 \mathrm{~m}, \sqrt{T / \mu}=1 \mathrm{~m} / \mathrm{s}$. Only look at the first five modes, that is, let $\mathrm{n}=5$.

Students are also asked to plot the frequency response of the vibrating string versus frequency in hertz using Bode plot conventions (that is, plot the magnitude in decibels and the frequency on a 
log scale). This system is very lightly damped, and all of the harmonics display high resonant peaks.

In class, the students must use a DAQ device and soft instrument panels on their laptops to display the measured signal in the time domain and frequency domain. The steps of the lab are

A. Time Domain Analysis (using an oscilloscope)

- Obtain the zero input response (initial condition response) and the impulse response and view the signals in the time domain.

o The zero input response is obtained by plucking the guitar string.

o The impulse response is obtained by hitting the string sharply with a screwdriver shaft.

- Understand what harmonics might look like in the time domain by measuring the fundamental frequency and identify the second harmonics in the time domain signal.

Figure 4 shows a portion of the impulse response. Students can see the effect of small damping values, and they can identify the small peak at the bottom of the curves as being due to the second harmonic.

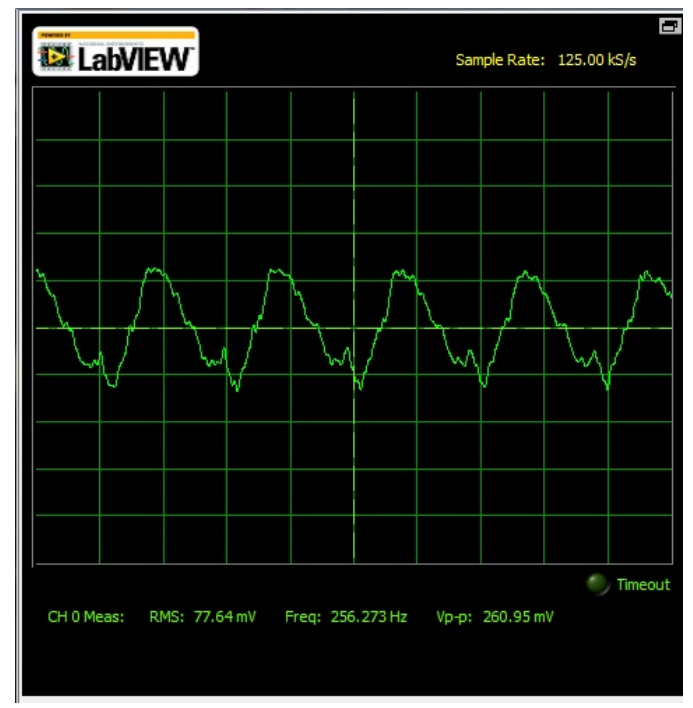

Figure 4: Zoomed in view of the impulse response.

B. Frequency Domain Analysis (using a spectrum analyzer)

- Examine the ambient signal, that is, without plucking or hitting the string, in order to identify noise in the measurements, such as a $60 \mathrm{~Hz}$ signal and its harmonics so that these elements can be ignored in the later measurements.

- Hit the string with an impulsive input and use the spectrum analyzer to plot the corresponding frequency response. From the spectrum, measure the fundamental frequency and frequency of the harmonics and compare to the values found from the time domain signals.

Figure 5 is a screen shot of the measured frequency spectrum. Notice the distinct harmonics. 


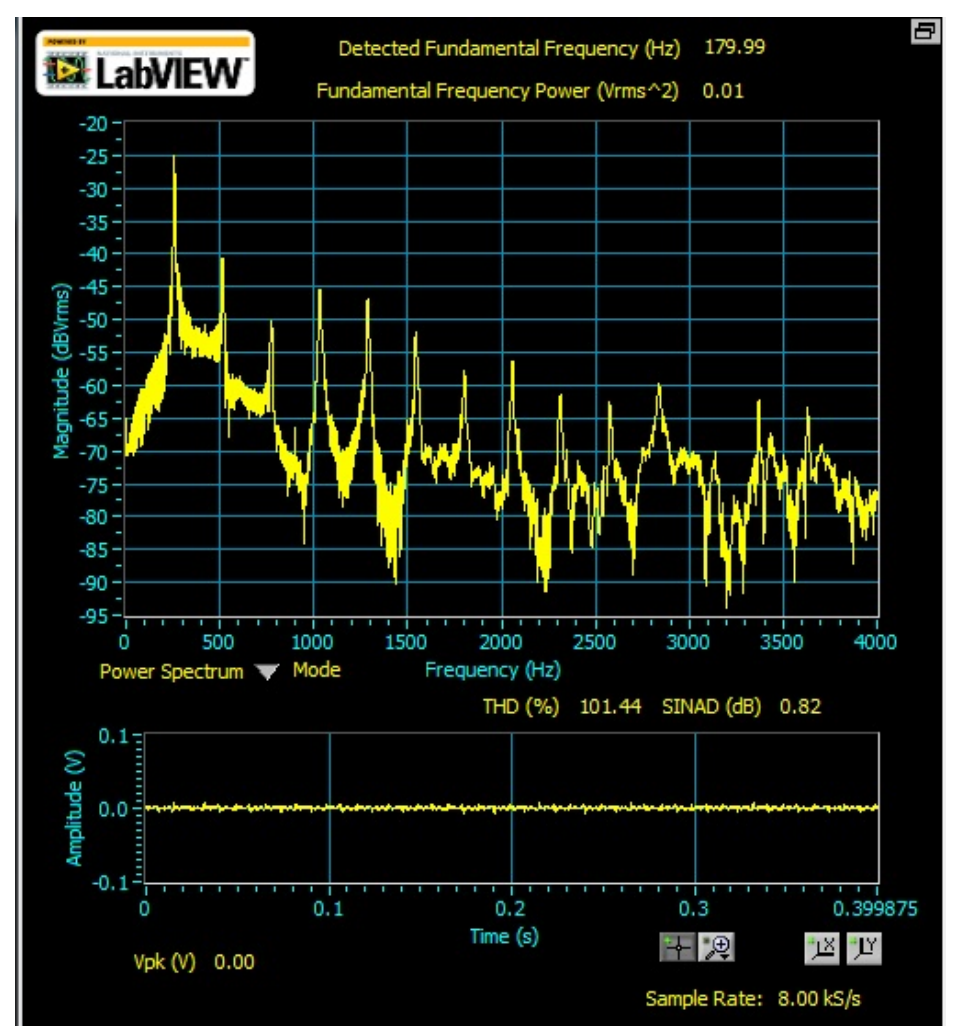

Figure 5: Magnitude of the frequency spectrum resulting from an impulsive excitation.

C. Examine controllability and observability properties

- Hit the string near one of the ends and compare both the sound and the spectrum to the response resulting from hitting the string exactly in the middle.

- Place the pick-up near the end to record the signal from an impulse applied near an end. Compare this signal to the result when the transducer is placed under a node.

D. Modify the System

- Use the system equations in the appendix to understand the system model and to see how the system can be modified to achieve desired responses.

- Tune the system by changing the tension in the string to a specific note. Use frequency response plot to help tune to the correct frequency.

- Obtain a desired tone by calculating where the fret must be placed to achieve that note, and verify the results.

E. Obtain the Frequency Response via a Sine Sweep

A sine sweep requires a sinusoidal vibratory input to the system, as is typical with a vibration shaker. Vibration shakers are fairly expensive, so a Rock-It ${ }^{\mathrm{TM}}$ speaker costing $\$ 25$ is used as a cheap shaker. This speaker, shown in Figure 6, has two main parts: the large shell containing the electronics and a small shell that contains a hard round plate that vibrates. This smaller shell is normally fastened to a large object in order to generate a louder sound than is achievable by the speaker alone. The USB cable is connected to a laptop to supply power, and the speaker input is connected to an analog output of the DAQ device supplying the sinusoidal signals. The hard plate is placed directly on the guitar string, and a sine sweep is run. Whenever the frequency 
goes through a harmonic, the sound gets much louder, the plotting spectrum reaches a peak magnitude value, and the amplitude of the measured signal increases dramatically.

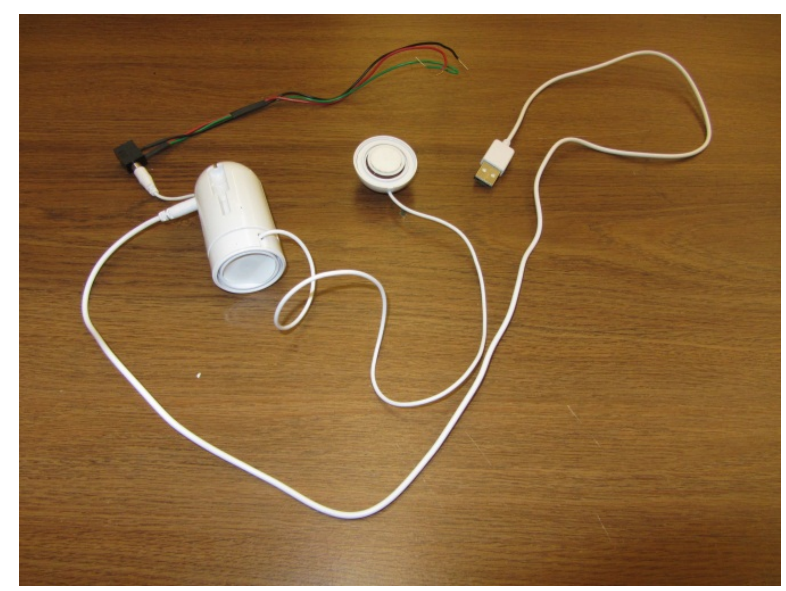

Figure 6: A Rock-It ${ }^{\mathrm{TM}}$ speaker used as a vibration shaker.

\section{Learning Objectives and Assessment}

While experiments have long been considered as central to engineering education, the specific learning outcomes have not always been clearly identified. Feisel and Rosa ${ }^{8}$ list 13 fundamental objectives of instructional laboratories. The portable experiment developed in this paper touches on many of these objectives and can be easily modified to emphasize some objectives vs others. The experiment gives students exposure to Instrumentation, Models, Experimentation, and Data Analysis (objectives 1-4 of Feisel and $\operatorname{Rosa}^{8}$ ). The use of a guitar string as the vehicle for learning allows the students to develop Psychomotor (the ability to actually touch and manipulate the device) and Sensory Awareness (objectives 8 and 12, respectively.) The exercise also helps reinforce "soft skills that are so important in professional practice: Safety, Communication, Teamwork, Ethics (objectives 9 -12). Tuning, fret positioning, the tone control circuit relate to Design (objective 5). Finally, although not intentional, students will sometimes get exposure to objective 6: Learning from Failure. The portability and affordability of experiment opens up the possibility of the reaching students in non-traditional settings, such as Massively Open Online Courses (MOOC's) as well as traditional asynchronous distance learning modes. Remote laboratories and virtual laboratories provide another way of reaching these students, but they are unable at this point to address objectives 8 and 13 (psychomotor and sensory awareness.)

The development and implementation of this particular experiment in the ECE 3084 Signals and Systems course at Georgia Tech is a direct result of a previous study where laboratories and experiments were inserted into another similar lecture-based course, ECE 3085 Introduction to Systems and Controls ${ }^{3,9}$. In ECE3085, students used LEGO NXT kits for three in-class and takehome projects that examined aliasing, digital filter implementation, and motor control. Assessment in that class included a concept inventory, student pre- and post-experiment surveys, and a survey taken one semester after the course ended. A control group consisted of a class section that did not use the experiments but worked extra problems while students in the other section did use the experiments. The assessment results are given in Ferri, et al. ${ }^{9}$ and briefly summarized here. Students who did the experiments performed better on the concepts inventory 
test than did the control group on questions that were related to the topics covered by the experiment. For example, a question that related to the purpose of a PI compensator resulted in 81.5\% correct in the experimental group while only $53.6 \%$ correct in the control group. Furthermore, a student survey taken one term later to assess the retention of knowledge showed marked improvement in student understanding due to the experiments. In particular, students were asked to rank their level of understanding of 11 topics in the ECE 3085 course that they took the previous semester, and $62 \%$ of students in the experimental group stated that they had a "solid understanding" of discretization of continuous-time systems compared to only $14 \%$ in the control group. Based on the demonstrated success of the use of the three experiments in ECE 3085, the faculty decided to incorporate experiments into the new ECE3084 course, and the guitar string experiment was designed and implemented along with two other experiments, one being the LabVIEW LEGO motor control experiment described in Ferri, et al. ${ }^{9}$ ported to the NI myDAQ.

\section{Summary}

The simple platform has been used in circuits classes and in K-12 outreach activities. The more complex platform has been used in two signals and systems classes in Fall 2012. Each class had approximately 50 students enrolled. The guitar string platforms were rolled into the classroom on a cart and distributed to the class, who formed two-person lab groups. The nominal lecture time was 50 minutes. The experiment as stated could not be done by the students in that time frame, so an instructor can eliminate or streamline various parts by doing that activity him or herself. In the Fall 2012 offering of the course, Part A and E were demonstrated by the instructor and the students completed the remainder of the steps themselves. A video of Part E is available online www-srv2.ece.gatech.edu/academic/Fall12/TESSAL/TESSAL_GuitarFreqResp.html. For logistics, it is a good idea for students to have a contingency plan of finishing the lab out of class during TA office hours in case they run out of time in class. Knowing the fall-back plan, students are less worried about completing the lab during class time and tend to be a little more exploratory in their approach. Very few students did not finish the lab on time. Preliminary analysis of student and instructor response to the guitar string experiment has been very positive, and follows the similar results as found in the previous study. The experiment will be used in two more classes in Spring of 2013.

Acknowledgement: Thanks to Dr. Don Millard, who has always found many ways to bring music to the classroom and, especially, the high school science teachers who built their own onestring guitars. Thanks to James Steinberg and Kevin Pham from Georgia Tech, who built the new versions of the experiment. This work was supported under NSF DUE-0717832 and NSF TUES 1226065. 


\section{Appendix: Equations of a Vibrating String}

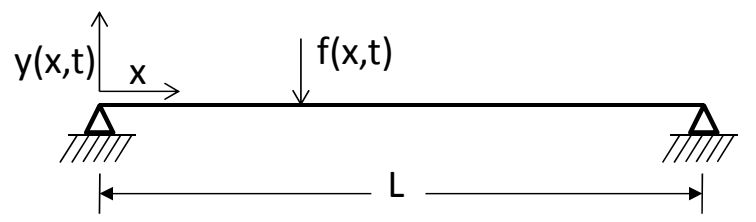

\begin{tabular}{|c|l|c|}
\hline \multicolumn{2}{|c|}{ Nomenclature } \\
\hline symbol & \multicolumn{1}{|c|}{ description } & units \\
\hline $\mathrm{L}$ & string length & $\mathrm{m}$ \\
\hline $\mathrm{T}$ & tension & $\mathrm{N}$ \\
\hline$\mu$ & mass/unit length & $\mathrm{kg} / \mathrm{m}$ \\
\hline $\mathrm{x}$ & position coordinate & $\mathrm{m}$ \\
\hline $\mathrm{xf}$ & location of applied inpulsive force & $\mathrm{m}$ \\
\hline $\mathrm{xr}$ & location of measured dispacement or velocity & $\mathrm{m}$ \\
\hline $\mathrm{t}$ & time & $\mathrm{s}$ \\
\hline $\mathrm{y}(\mathrm{x}, \mathrm{t})$ & string displacement at location $\mathrm{x}$ and time $\mathrm{t}$ & $\mathrm{m}$ \\
\hline $\mathrm{f}(\mathrm{x}, \mathrm{t})$ & external force/unit length & $\mathrm{N} / \mathrm{m}$ \\
\hline
\end{tabular}

Using the quantities defined in the figure above, the vibration of the string is modeled as a one dimensional wave equation:

$$
T \frac{\partial^{2} y}{\partial x^{2}}-\mu \frac{\partial^{2} y}{\partial t^{2}}=f(x, t)
$$

Note that this partial-differential equation has both space and time dependence. The system parameters can be in any consistent units, but the Nomenclature Table above defines the terms in standard SI units.

\section{Approximation to ordinary differential equations}

The free response of this system has characteristic shapes called "modes of vibration" as seen in Figure 3.

The equations describing the modes are given by

$$
\varphi_{k}(x)=\sqrt{\frac{2}{\mu L}} \sin (k \pi x / L)
$$

where the scaling term is used to normalize the resulting ordinary differential equations given below $^{10}$. The first mode corresponds to $\mathrm{k}=1$, second mode to $\mathrm{k}=2$, etc. Approximate $\mathrm{y}(\mathrm{x}, \mathrm{t})$ as a superposition of these modes,

$$
y(x, t)=\sum_{k=1}^{n} q_{k}(t) \varphi_{k}(x)
$$


where $\mathrm{q}_{\mathrm{k}}(\mathrm{t})$ represents an amplitude of the mode shape as a function of time. It represents the contribution of that mode shape to the response of the system. If the system is forced at a node of a mode shape, then $\mathrm{q}_{\mathrm{k}}(\mathrm{t})=0$ for that mode shape.

This expression is substituted into the wave equation for $\mathrm{y}$, and assume that the force is applied at a single point $\mathrm{x}=\mathrm{x}_{\mathrm{f}}$, so $(x, t)=f\left(x_{f}, t\right) \equiv \delta\left(x-x_{f}\right) u(t)$, where $\delta$ represents a spatial Dirac delta function. A lot of simplification is done here in order to obtain a set of ordinary differential equations that are used to model the system:

$$
\ddot{q}_{k}+2 \xi \omega_{k} \dot{q}_{k}+\omega_{k}^{2} q_{k}=\varphi\left(x_{f}\right) u(t) \quad \text { for } k=1, n
$$

where $\xi$ is the damping ratio, often taken to be 0.01 for lightly damped systems such as this string and $\omega_{\mathrm{k}}$ is the natural frequency of the $\mathrm{k}^{\text {th }}$ mode in rad/sec. The natural frequencies can be shown to be given by

$$
\omega_{k}=\frac{k \pi}{L} \sqrt{\frac{T}{\mu}}
$$

To find the output measured as a single point, $x=x_{r}$, use the series form for $y(x, t)$ :

$$
y(t) \equiv y\left(x_{r}, t\right)=\sum_{k=1}^{n} q_{k}(t) \varphi_{k}\left(x_{r}\right)
$$

The frequency response of this system can be found by taking the Fourier Transforms of the $n$ second-order differential equations and that of the output signal and solving for $Y(j \omega) / U(j \omega)$. In this system, the guitar pickup actually measures velocity of the moving wire, $\dot{y}(t)$, not the displacement, $y(t)$, at the guitar pickup point $x_{r}$ So we ultimately want $H(j \omega)=\dot{Y}(j \omega) / U(j \omega)$.

Taking the Fourier transform of both sides of the above ordinary differential equation and using the derivative property of Fourier transform yields:

$$
\begin{gathered}
(j \omega)^{2} Q_{k}(j \omega)+2 \xi \omega_{k}(j \omega) Q_{k}(j \omega)+\omega_{k}^{2} Q_{k}(j \omega)=\varphi\left(x_{f}\right) U(j \omega) \quad \text { for } k=1, n \\
Q_{k}(j \omega)\left[(j \omega)^{2}+2 \xi \omega_{k}(j \omega)+\omega_{k}^{2}\right]=\varphi\left(x_{f}\right) U(j \omega) \quad \text { for } k=1, n
\end{gathered}
$$

Taking the Fourier transform of both sides of the above Fourier sum and applying the derivative property of Fourier transforms once more yields:

$$
\dot{Y}(j \omega)=j \omega \sum_{k=1}^{n} Q_{k}(j \omega) \varphi_{k}\left(x_{r}\right)
$$

From our last two equations, the frequency response function $H(j \omega)=\dot{Y}(j \omega) / U(j \omega)$ is obtained. 


\section{References}

1. Engineering and the Guitar, Agilent Technologies, http://www.home.agilent.com/agilent/editorial.jspx?cc=AW\&lc=eng\&ckey=866697\&id=866697, downloaded $1 / 7 / 13$

2. K. Connor, F. Berry, M. Chouikha, D. Newman, M. Deyoe, G. Anaya, W. Brubaker. "Using the Mobile Studio to Facilitate Non-Traditional Approaches to Education and Outreach,” ASEE Annual Conference, Vancouver, BC, June 2011 AC2011-2250

3. B. Ferri, S. Ahmed, J. Michaels, E. Dean, C. Garyet, S. Shearman, "Signal Processing Experiments with the LEGO MINDSTORMS NXT Kit for Use in Signals and Systems Courses," Proceedings of the 2009 American Control Conference, St. Louis, MO, June 2009.

4. B. Ferri, J. Auerbach, J. Michaels, and D, Williams, “TESSAL: A Program for Incorporating Experiments into Lecture-Based Courses within the ECE Curriculum,” ASEE Annual Conference and Exposition, Vancouver, Canada, June 2011.

5. K. Meehan, R.W. Hendricks, R.L. Clark, C. Shek, "Lab in a Box: The development of materials to support independent experimentation on concepts from circuits," in Proc. 2009 ASEE Annu. Conf. Expo., Austin, TX, 2009, AC 2009-411.

6. Y. Xu, K. Meehan, C.V. Martin, A.B. Overby, and X. Wei, "Visualizing concepts in electromagnetic fields: Hands-on experiments using student-owned laboratory kits," in Proc. 2011ASEE Annu. Conf. Expo., Vancouver, BC, 2011, AC 2011-1682.

7. NI myDAQ, http://www.ni.com/mydaq/, downloaded 1/7/2013.

8. Feisel and Rosa, 2005, “The Role of the Laboratory in Undergraduate Engineering Education,” Journal of Engineering Education, Jan., pp. 121- 130.

9. G. Droge, B. Ferri, J. Auerbach, "Distributed Laboratories: Control System Experiments with LabVIEW and the LEGO NXT Platform,” ASEE Annual Conference and Exposition, San Antonio, TX, June 2012.

10. J.H. Ginsberg, 2001, Mechanical and Structural Vibrations: Theory and Applications,” John Wiley and Sons, NY. 